\title{
D'où venons-nous ? Que sommes-nous ? Où allons-nous ?
}

\section{Where do we come from? Who are we? Where are we going?}

\section{Claudine Friedberg}

Anthropologue, Département Hommes, natures, sociétés, Muséum national d'histoire naturelle, 57 rue Cuvier, 75231 Paris cedex 05, France

Régulièrement, les médias nous informent que les paléontologues ont découvert de nouveaux fossiles qui infirment les hypothèses antérieures sur l'origine et l'histoire de nos ancêtres. Dans le même temps, ces mêmes médias nous laissent entendre que nos différences sur le plan génétique avec nos parents animaux les plus proches, les grands singes, tiendraient à très peu de choses. La possibilité de faire entrer dans le genre Homo le chimpanzé, pour lequel cette différence se réduit à moins de $1 \%$, a même eu les honneurs d'un journal télévisé de 20 heures $^{1}$. Tout ceci révèle une inquiétude sur ce que nous sommes et sur nos relations avec le reste du monde vivant. Où et quand apparaît notre lignée ? À partir de quand peut-on parler d'humains, c'est-à-dire comment les identifier ? En quoi consiste notre originalité ? S'il est devenu indécent de reconnaître dans l'espèce humaine l'existence de races définies biologiquement, la possibilité d'une origine polyphylétique des hommes actuels, à partir des différentes espèces d'hommes ayant existé dans le passé, ressurgit périodiquement.

À une époque où l'explosion et l'exposition au grand jour de ce qui apparaît à la plupart comme des déviances « non humaines », où la violence individuelle ou collective, qu'elle soit le fait des

\footnotetext{
Allusion au tableau ainsi intitulé et peint par Gauguin en 1887, dont on trouvera une reproduction p. 489 du deuxième volume de Y. Coppens et P. Picq (2001).

Adresse e-mail : Friedbg@mnhn.fr (C. Friedberg).

1 Journal télévisé de France 2 le 26 mai 2003.
}

États, des groupes politiques, économiques ou confessionnels, semblent devenir la règle de vie pour l'humanité entière, les interrogations sur nous-mêmes se multiplient, et elles ne peuvent faire fi de ce que la science nous apprend sur l'histoire et les caractéristiques de notre espèce et de ses ancêtres.

Les ouvrages dans ce domaine sont nombreux et les hypothèses qu'ils proposent peuvent apparaître contradictoires. Même s'ils ne fournissent pas de réponses définitives, les deux ouvrages dirigés par $\mathrm{Y}$. Coppens et $\mathrm{P}$. Picq ${ }^{2}$ serviront de base à mes réflexions. Ils ont l'avantage de réunir des textes écrits par des spécialistes appartenant à plusieurs disciplines. Or, cette confrontation d'informations issues de domaines différents semble nécessaire pour éclairer les questions posées et renouveler les problématiques, en réunissant les données issues de l'archéologie et de la paléontologie (dans le premier volume) avec celles découlant de l'observation sur le vivant (dans le second).

On peut regretter que les éditeurs scientifiques, en plaçant le lecteur face à des hypothèses multiples, l'abandonne dans une grande incertitude en lui laissant le soin de recouper lui-même les informations. On trouvera dans ce même numéro un

${ }^{2}$ Aux origines de l'humanité, sous la direction de Yves Coppens et Pascal Picq. Préface de Yves Coppens. Projet scientifique conçu par Pascal Picq, Paris, Fayard, 2001. Tome 1 : De l'apparition de la vie à l'homme moderne, $650 \mathrm{p}$.; Tome 2 : Le propre de l'homme, $569 \mathrm{p}$. 
compte rendu principalement consacré aux données matérielles (contenues dans le premier volume et dans trois chapitres du second) à partir desquelles les scientifiques élaborent leurs hypothèses sur l'origine de l'humanité et le « propre de l'homme ", jusqu'à l'apparition et la dispersion du genre Homo. Mon objectif est ici de reprendre l'histoire à ce moment-là, en examinant principalement ce qui touche à l'aspect social et cognitique des hypothèses émises sur nos ancêtres et sur nousmêmes.

Cependant, en préambule, il me paraît indispensable de dire un mot des théories de l'évolution qui sous-tendent, souvent de façon implicite, les raisonnements des uns et des autres.

\section{Les théories de l'évolution et l'apparition de l'homme}

P. Picq évoque l'influence tenace sur la paléontologie de la théorie synthétique de l'évolution défendue par Ernst Mayr, qui correspond à une vision gradualiste de l'émergence de l'homme à travers une suite d'espèces entre lesquelles il est difficile d'établir des frontières. L'autre théorie invoquée est celle des équilibres ponctués, c'est-à-dire une évolution qui procède par sauts, séparés par des périodes de stabilité. En outre, les spécialistes ont adopté une méthode d'analyse de l'histoire phylétique appelée cladistique, dans laquelle on repère les phénomènes de spéciation, ou cladogénèse, en mettant en évidence les caractères dérivés. Ceci permet de construire des arbres phylogénétiques à partir des relations entre les taxons (élément de classification à n'importe quel niveau hiérarchique) sans avoir à identifier d'ancêtre commun. Deux espèces ou deux genres sont d'autant plus proches qu'ils partagent un plus grand nombre de caractères dérivés ou évolués.

Alors que sont souvent évoqués, pour expliquer l'apparition de nouvelles formes, des phénomènes d'adaptation aux modifications du milieu et aux ressources alimentaires disponibles, P. Picq rappelle utilement que « l'environnement ne crée rien, il ne fait que sélectionner ». Cependant, selon la théorie de l'évolution que l'on adopte, le mécanisme de cette sélection n'est pas le même, et l'on perçoit parfois chez certains auteurs des relents de néo-lamarckisme. Encore qu'il faille peut-être rester prudent dans ce domaine, ce que l'on appelle l'hérédité épigénétique pouvant nous réserver des surprises. Il semble bien que l'expression des gènes dépende du contexte. Or, une petite modification de l'ADN peut entraîner une grande différence dans les protéines produites, ce qui pourrait expliquer les « sauts » évolutifs.
La faible diversité des hominoïdes existant encore aujourd'hui (les gibbons, les orangs-outans, les gorilles, les chimpanzés et les hommes) contraste avec ce qu'elle fut au début du Miocène, où se développèrent plusieurs grandes lignées. L'émergence de l'homme correspond au déclin des grands singes hominoïdes : il est la dernière espèce de sa lignée évolutive, celle des homininés. La multiplication des découvertes de restes fossiles, au lieu d'éclaircir le problème de nos origines, le rend encore plus obscur. Elles confirment la nonlinéarité de notre évolution : elle ne s'est pas effectuée en trois étapes, un grand singe ancestral donnant naissance aux australopithèques puis aux hommes. Cette évolution est « buissonnante, comme celle de toutes les autres lignées à un moment ou un autre de leur histoire évolutive » (P. Picq, p. 573).

Comme le dit B. Senut, le « passage des grands singes hominoïdes ancestraux aux premiers représentants de la lignée humaine n'est pas aisé à déterminer [...] il n'existe pas de caractère magique permettant de trancher : on est en présence d'un faisceau de caractères à replacer dans un contexte qui doit inclure toutes les données disponibles » issues de plusieurs disciplines.

On a fait ainsi appel à la biologie moléculaire, et les travaux sur l'ADN et les chromosomes ont mis en évidence la proche parenté entre les chimpanzés (chimpanzé commun et bonobo) et l'homme. Quant au concept « d'horloge moléculaire » fondée sur le renouvellement de l'ADN au cours du temps, dont on espérait qu'il permettrait de dater les différentes dichotomies de la phylogénie des grands singes et de l'homme, il n'a pas donné les résultats escomptés, la question étant plus complexe que ce que l'on imaginait. En effet, les « horloges » ne battent pas au même rythme pour des gènes différents d'un même chromosome, dans une même espèce pour l'ADN chromosomique et mitochondrial et dans des espèces différentes d'un même lignage.

\section{De l'émergence du genre Homo à celle de l'homme moderne ${ }^{3}$}

Depuis un million d'années notre lignée n'est plus représentée que par le genre Homo.

Les capacités d'adaptation et la grande mobilité des groupes sont sans doute à l'origine de la dispersion des premiers hommes à l'extérieur de l'Afri-

\footnotetext{
${ }^{3}$ Nous nous appuyons ici sur les chapitres rédigés par JeanJacques Hublin et par Bernard Vandermeersch.
} 
que, en particulier au sud de l'Eurasie, comme l'indiquent les fossiles trouvés en Georgie et datant d'environ 1,8 million d'années. Ne pouvant déterminer à quel type d'homme connu appartiennent des restes découverts depuis la parution de l'ouvrage, on l'a baptisé $H$. georgicus. Des fragments datant à peu près de la même époque ont été trouvés en Chine et à Java. S'agit-il d'H. ergaster qui se serait déplacé très rapidement après son émergence, ou d'une espèce d'homme apparue antérieurement ? Les paléontologues hésitent. À partir de quand peut-on parler d'Homo erectus ? Là aussi les hypothèses divergent. Pour certains, ce concept doit être limité aux populations d'Asie. Pour d'autres, $H$. erectus qui a vécu sur une période allant de 1,8 million d'années à 300000 ans est l'ancêtre d' $H$. sapiens, alors que pour d'autres encore les précurseurs des hommes modernes et des Néanderthaliens sont à rechercher parmi les quelques restes d'hominidés africains datant de 1,3 million à 600000 ans.

Vers 500000 ans, on constate une modification du rythme d'alternance des périodes glaciaires et interglaciaires et l'accroissement de leur amplitude. Les hommes se sont déplacés au gré des avancées et des reculs des glaciers et du déplacement des rivages marins. Certaines populations se sont trouvées isolées ; c'est le cas des Néanderthaliens en Europe, dont l'émergence est à mettre en relation avec les conditions climatiques et géographiques. Leurs industries furent d'abord l'Acheuléen puis le Moustérien, caractérisé par ses outils sur éclats, apparu vers 200000 ans et qui a duré jusqu'à il y a 30000 dans le sud de la péninsule ibérique. Cependant, une part importante de l'outillage confectionné en bois ne nous est pas parvenu. L'art est absent de cette culture, mais non la décoration, comme en témoignent les colorants trouvés dans les gisements et des tracés gravés sur os. Des sépultures ont aussi été découvertes.

Pendant ce temps, l'homme moderne, Homo sapiens, fait son apparition en Afrique. La morphologie moderne émerge vers 150000 ans en Afrique orientale, autour de 100000 en Afrique du Sud et sans doute un peu plus tard au Maroc. Au Levant, on a découvert plusieurs squelettes à morphologie moderne, dans des niveaux moustériens (datés entre 105000 et 95000 ans). Ces restes sont-ils ceux de populations ayant évolué sur place ou ceux de migrants venant d'Afrique ? Difficile de répondre car on ne note aucune rupture dans les cultures locales.

En Asie du Sud-Est, l' $H$. sapiens archaïque est mal connu. Quant aux hommes modernes, ils ne sont représentés que par des restes récents. Pour ce qui est de l'arrivée d'H. sapiens en Australie, elle date de plus de 60000 ans, ce qui laisse supposer qu'il possédait déjà le moyen de s'aventurer sur la mer, car, même aux périodes où le niveau de la mer a été le plus bas, le détroit séparant le continent de Sunda de celui de Sahul n’a jamais été inférieur à $70 \mathrm{~km}$. Le peuplement de l'Australie s'est fait en une ou deux vagues à partir de populations antérieures à celles vivant actuellement en Asie du Sud-Est.

En Asie continentale, on aurait trouvé des formes de transition entre les $H$. erectus chinois et les $H$. sapiens d'un âge compris entre 100000 et 125000 ans, mais les conditions de découverte des restes ne permettent malheureusement de dater de façon sûre que ceux dont l'âge ne dépasse pas 20000 ou 30000 ans.

En Europe, l'homme moderne est arrivé par l'est, il y a environ 40000 ans, et l'on ne trouve aucune trace de morphologie de transition à partir des Néanderthaliens. Les deux populations ont coexisté pendant une durée pouvant aller jusqu'à plusieurs millénaires dans certaines régions. Il y a sans doute eu des échanges sur le plan culturel, mais nous n'avons aucune preuve de métissage. Peu à peu, les Néanderthaliens vont laisser la place aux hommes modernes et vont inexorablement disparaître, non pas parce que leurs capacités cognitives sont moindres, mais peut-être parce qu'ils étaient biologiquement, et par leur mode de vie mobile, adaptés aux fluctuations climatiques et aux déplacements du gibier dont ils se nourrissaient. Quand les hommes modernes sont arrivés, les Néanderthaliens ont retrouvé certains de leurs habitats occupés et ont dû chercher refuge ailleurs. À partir de 30000 ans, il ne reste plus qu'une seule sousespèce, Homo sapiens sapiens.

La différence entre la mise en place de l'humanité moderne en Europe et dans le reste du monde nous laisse présager qu'elle est le résultat d'un processus complexe faisant intervenir des évolutions, des remplacements, des migrations, dans un ensemble de populations entre lesquelles ont pu se produire des flux géniques.

\section{L'homme moderne a-t-il une origine unique?}

Trois scénarios nous sont proposés par Véronique Barriel. D'abord le modèle candélabre ou multirégional, dans lequel l'Homo sapiens apparaît indépendamment en plusieurs régions du monde à partir de formes archaïques dans les régions colonisées par $H$. erectus entre 1,7 et 0,7 million d'années. Mais il est difficile d'admettre qu'une même es- 
pèce apparaisse simultanément en plusieurs endroits. Dans le deuxième modèle, out of Africa, l'homme moderne a son origine en Afrique subsaharienne, d'où il se serait répandu dans l'ancien monde entre les années 100000 et 400000 , remplaçant les populations existantes d' $H$. erectus et d' $H$. sapiens archaïque, la contribution génétique de ces populations à l'homme moderne étant négligeable. Le nombre d'ancêtres au moment de l'expansion démographique serait faible : hypothèse associée à l'idée d'un « goulot d'étranglement » sur la dimension duquel les chercheurs ne s'entendent pas, entre 100000 et 10000 individus. Enfin, on peut admettre des scénarios intermédiaires : l'homme moderne évolue d'abord en Afrique, puis sa dispersion sur d'autres continents s'accompagne de phénomènes d'hybridation.

Au début des années 80 , il devint possible d'étudier directement le polymorphisme de l'ADN. Des mesures faites à travers le monde montrent que la variabilité génétique entre les individus d'une même population s'élève entre 80 et $85 \%$, alors qu'elle n'est que de 10 à $15 \%$ entre populations différentes, ce qui invalide définitivement l'idée d'une base génétique du concept de race.

Tous les essais de reconstruction phylogénétique pour tenter de situer et de dater les divergences entre les clades se sont heurtés au fait que seuls des fragments d'ADN ont pu être étudiés. Les travaux sur de l'ADN mitochondrial vont dans le sens d'une origine africaine de l'homme moderne, avec une sortie d'Afrique entre 200000 et 100000 ans. Ceux sur le chromosome masculin $Y$ indiqueraient qu'il y aurait eu un retour en Afrique avec une nouvelle mutation du chromosome $Y$ apparu en Asie. Ajoutons qu'on ne peut assimiler généalogie de gènes et généalogie d'individus.

Depuis 1997, on a tenté des analyses et des séquençages de l'ADN de l'Homme de Néanderthal, dans des conditions très strictes et avec de nombreux contrôles. Ces études indiquent que la diversité néanderthalienne n'est pas beaucoup plus élevée que celle de l'homme actuel $(3,73 \%$ contre $3,43 \%$ ), alors qu'elle est de $14,82 \%$ chez les chimpanzés et de $18,57 \%$ chez les gorilles. Cette baisse de la diversité génétique humaine comme résultat d'une expansion rapide, à partir d'une population de taille réduite, s'appliquerait donc aussi aux Néanderthaliens.

Pour l'instant les données moléculaires semblent privilégier l'hypothèse d'une origine africaine de l'homme moderne, avec un remplacement rapide des populations antérieures dans tout l'Ancien Monde. Mais cela s'est passé d'une façon certainement beaucoup plus complexe que nous ne l'imagi- nons, avec des mouvements migratoires multiples et dans des directions variées entraînant des mélanges géniques dans lesquels les femmes, plus mobiles que les hommes, auraient joué un rôle important.

L'installation de l'homme moderne sur l'ensemble de la planète est partout marquée par des manifestations artistiques (statuettes, gravures et peintures rupestres) qui sont des témoignages précieux des capacités cognitives de nos ancêtres et de leur volonté de communiquer : idéogrammes, pictogrammes, figures plus ou moins naturalistes ou stylisées manifestant plus un moyen d'expression qu'une volonté de représentation d'une réalité.

Je ne reprendrai pas ici les différentes hypothèses émises sur le déchiffrement et le pourquoi de ces expressions artistiques. Je ne formulerai qu'une seule remarque sur la façon dont $\mathrm{E}$. Anati, auteur du chapitre consacré à ce sujet, met l'accent, à plusieurs reprises, sur les similitudes dans les phases initiales des expressions artistiques à travers le monde, des différences de style n'apparaissant qu'entre 16000 à 12000 ans. Pour l'auteur, ces similitudes traduisent «la présence d'une même logique et un même système d'association d'idées ", que l'on peut mettre en relation avec une vision dualiste du monde opposant deux éléments complémentaires dont le prototype est le masculin et le féminin et qui « laisse envisager l'existence d'une langue mère universelle ».

Tout ce que l'on peut dire, c'est qu'effectivement l'homme, où qu'il soit, dispose, à partir de ce qu'il observe dans son environnement, d'un choix limité d'éléments pour ordonner et se représenter le monde (le féminin / le masculin, le jour / la nuit, le haut / le bas, le ciel / la terre, etc.), qu'il les associe deux à deux en les opposant ou en insistant sur leur complétude. Les similitudes viennent de ce que les thématiques et les modes d'expression sont restreints, mais, de là à envisager qu'il y aurait eu pendant ces millénaires d'apprentissage de l'expression parlée une langue mère universelle, il y a un grand pas qu'il faut se garder de franchir. Si l'on peut imaginer qu'au départ, le langage articulé est né dans le petit groupe où est apparu la transformation anatomique qui le permettait, très vite, au fur et à mesure de la diffusion de celle-ci dans des groupes différents, des langues et des cultures associées se sont structurées tout en se diversifiant. Même en faisant l'hypothèse d'un goulot d'étranglement de 10000 individus pour les premières populations d'Homo sapiens, ce que l'on peut concevoir de la taille des groupes à cette époque plaide pour une diversité des langues utilisées. 


\section{Comment repérer dans les comportements ce qui est propre à l'homme et ce qu'il partage avec d'autres animaux : évolution, sélection, comparaison?}

Les paléontologues et les archéologues qui ont collaboré au premier volume n'hésitent pas à émettre, à partir d'indices matériels souvent ténus, des hypothèses sur le développement cognitif et les comportements de nos ancêtres, mais c'est dans le deuxième volume que cette question est véritablement traitée en cherchant à définir le « propre de l'homme ". Les différents auteurs ${ }^{4}$ formulent alors leurs hypothèses et conclusions à partir d'observations faites sur le vivant, dans la nature ou lors d'expérimentations. Ces recherches sont récentes. Dans les années 1950-1960, les Japonais établissent les fondements de l'éthologie moderne, alors que les primatologues occidentaux en sont encore à décrire des systèmes sociaux régis par l'agressivité et la violence des mâles. Les travaux de terrain sur le comportement des chimpanzés comme ceux de J. Goodall ont débuté il y a une trentaine d'années, précédant de peu les recherches de laboratoire de A. et B. Gardner et celles de D. Premack.

Cependant, pour interpréter ces observations, la lecture préalable de la conclusion de P. Picq n'est pas inutile. Il y relève, en particulier, que « l'apport de la révolution cladistique - ou systématique phylogénétique $-[. .$.$] consistant à reconstituer les$ relations de parenté entre lignées [...] n'est pas encore bien mesuré ». Il ajoute : "Resituer le propre de l'homme ne consiste pas à opérer un tri ni un amalgame entre toutes les observations disponibles comparant l'homme et les autres espèces mais à les ordonner en fonction [...] des relations phylogénétiques entre les espèces concernées ».

Or, pour tenter de cerner "le propre de l'homme ", les données fournies par les différents auteurs ne sont pas toujours concentrées sur les espèces phylétiquement les plus proches, les chimpanzés et les bonobos, mais elles concernent aussi d'autres primates très éloignés ou d'autres mammifères et jusqu'à des oiseaux. Cela n'est pas complètement sans intérêt dans la mesure où nous constatons ainsi la diversité des comportements animaux, mais il ne faudrait pas que ces exemples faussent

\footnotetext{
${ }^{4}$ En dehors de ceux cités dans le compte rendu (voir dans ce même numéro), ces auteurs sont dans l'ordre de leur intervention : Jan A.R.A.M. Van Hooff, Bertrand L. Deputte et Jacques Vauclair, Dominique Lestel, James R. Anderson, Frans Waal et Bernard Thierry, Boris Cyrulnik, Élisabeth de Fontenay et à nouveau Pascal Picq pour la conclusion.
}

notre vision des possibilités qui s'offrent à l'humanité compte tenu de sa situation phylétique. On ne dira jamais assez combien les manifestations d'agressivité des oies de Lorentz auront hypothéqué durablement notre compréhension de l'homme. Même si l'agression fait partie de notre registre comportemental, c'est au même titre que les attitudes d'apaisement et de réconciliation.

De la même façon, le comportement de rats enfermés à titre expérimental en surnombre dans une cage, et qui se sont entretués, ne peut être transposé à l'homme. Par contre, des chimpanzés mis dans un espace restreint évitent de provoquer des conflits et s'efforcent de ne pas engager de contacts visuels ou physiques, ce qui évoque ce que l'on appelle le " comportement de l'ascenseur » chez l'homme.

La conclusion de P. Picq remet bien des choses en place par rapport à ce qui est dit dans les différents chapitres et nous entraîne souvent, sous le prétexte de réfuter des hypothèses erronées, vers de faux problèmes. Ainsi, prenons les chapitres rédigés par Van Hoof. L'un, destiné à démontrer, à partir d'observations chez le singe et l'homme, que le rire et le sourire ont une histoire évolutive différente, est très convaincant. Le rire a évolué à partir d'un signal d'intention de mordre dans un contexte de jeu ; tandis que l'origine de l'exposition des dents manifestée dans le sourire et traduisant chez les primates non-humains la crainte et la terreur, devient chez l'homme caractéristique d'une attitude apaisante ou d'excuse. D'une mimique de soumission craintive, on est passé à une expression de non-hostilité et d'amitié. Cependant, chez certaines espèces, la distinction semble se brouiller et il en est de même chez l'homme où rire et sourire peuvent participer d'interactions sociales complexes.

Par contre, le chapitre du même auteur intitulé "Vivre en groupe » pose de nombreuses questions sur les interprétations de ce qu'il appelle les «stratégies socio-sexuelles ». Les observations qui nous sont rapportées s'égarent entre de multiples espèces de primates pour expliquer la tendance à la vie en groupe chez la plupart des primates diurnes et ses différentes modalités. En fait, il est difficile de parler de «stratégies", ce qui impliquerait une volonté consciente, et l'auteur aurait dû s'en tenir à cette remarque : " l'évolution sélectionne un animal porté à développer des relations avec d'autres qui lui permettront [...] d'obtenir un maximum de descendants ".

De même, lorsqu'il se demande si l'apparentement génétique conduit à renforcer les liens sociaux et les comportements altruistes, il est pour le moins curieux qu'il ajoute que « cela ne signifie pas 
[...] que les individus perçoivent directement cet apparentement », ce dont personne ne doute. L'explication donnée plus loin se suffit à elle-même. Lorsque «la vie en groupe » s'organise entre parents, ceux-ci auront tendance à s'entraider ; c'est le cas des communautés de chimpanzés où les mâles restent dans le groupe natal, et cela n'a aucun rapport avec une quelconque conscience d'apparentement génétique! Ce renversement de perspective est d'autant plus dangereux qu'il est passé dans les médias grand public et que l'on entend des commentateurs de télévision déclarer devant l'image d'un accouplement entre une lionne et un lion que ce dernier est en train de transmettre ses gènes ! La mise en avant de la volonté de supprimer les gènes d'un autre mâle pour expliquer les infanticides des petits issus d'une copulation antérieure avec un autre, participe des mêmes erreurs de raisonnement.

L'exposé de la concurrence entre les mâles pour l'accès aux femelles est pour le moins confus. On en retiendra qu'il y a une corrélation entre l'accentuation du dimorphisme sexuel et le fait que des mâles dominants monopolisent les femelles. Dans le premier volume où cet aspect est traité, il est dit que ce dimorphisme encore très marqué chez les hominidés fossiles, et en particulier chez les australopithèques (à tel point que des restes attribués à des espèces différentes pourraient en réalité n'être que les formes mâle et femelle de la même espèce), est déjà fortement atténué chez $H$. ergaster. Se pose aussi la question de la visibilité de l'œstrus, dont on ne peut évidemment trouver trace dans les formes fossiles. Très voyant chez les chimpanzés et les bonobos, il a complètement disparu chez la femme.

Pourquoi faut-il encore là, en arriver à la conclusion de P. Picq pour voir exposer clairement que les singes, comme la plupart des mammifères, connaissent une exogamie de l'un des deux sexes, le plus souvent pour les mâles. Alors que chez les chimpanzés et les bonobos, ce sont les femelles qui quittent leur groupe natal. Ce comportement, corroboré par le très faible taux d'accouplement incestueux observé chez les chimpanzés, a été sélectionné au cours de l'évolution des mammifères, et l'évitement de l'inceste n'est donc pas une caractéristique de notre espèce.

Pour B. Cyrulnik, neuropsychiatre, le mécanisme provoquant l'évitement de l'inceste s'explique par un ensemble comportemental et émotionel lié à « l'attachement » mère-enfant qui se construit dans la petite enfance et, d'une façon générale, à «l'empreinte » que l'on garde du milieu écologique et affectif dans lequel on a été élevé, empêchant l'apparentement sexuel avec les êtres qui y parti- cipaient. Cet héritage phylogénétique a été transformé par l'homme en des règles sociales édictées sous des formes variables selon les sociétés.

\section{Capacités cognitives et apprentissage chez les humains et les non-humains}

Que reste-t-il du «propre de l'homme » après la lecture de ces deux ouvrages ? Le langage articulé et lui seulement, mais avec cette réserve qu'au moment où il est apparu, tout était déjà en place sur le plan cérébral pour qu'il puisse se manifester. L'aire de Broca et celle Wernicke existent chez le chimpanzé ; les moulages endocrâniens montrent que la première au moins existait déjà chez les hominidés vers 2 millions d'années. En outre, les structures cérébrales asymétriques indiquent qu'une même latérisation fonctionnelle préexiste chez les primates anthropoïdes (chimpanzés, gorilles, ourang-outans) avant l'apparition du langage articulé. Cependant, on peut retenir la formule de Cyrulnik, qui considére que la transformation anatomique entraînant la descente du larynx chez l'homme et permettant une communication d'un autre type conduit à une véritable métamorphose.

Plusieurs chapitres ayant pour auteurs B. Deputte, J. Vauclair, D. Lestel et J.R. Anderson, traitent, en combinant les apports de la psychologie expérimentale et de la primatologie, de la comparaison entre les capacités cognitives animales, surtout celles de primates non-humains, et celles de l'homme. Ainsi, des comportements de catégorisation ont été mis en évidence par des expériences chez divers animaux, en particulier sur des pigeons, révélant un niveau d'abstraction dans la reconnaissance d'arbres d'allures différentes. Des tests effectués sur des babouins ont montré leur aptitude à former une catégorie abstraite en classant des objets considérés comme « nourriture » ou « nonnourriture ». En outre, on constate chez les primates un développement important des régions du cerveau impliquées dans la perception et le traitement des informations visuelles.

Par ailleurs, de nombreuses expériences ont été mises en place pour prouver que les chimpanzés, qui ne possèdent pas d'appareil phonatoire, peuvent avoir accès à un langage. Certains protocoles ont été appliqués en considérant les cobayes comme des animaux de laboratoire, tandis que, pour d'autres chercheurs, l'apprentissage n'était possible que si le jeune primate était traité comme un enfant. Dans certains cas, l'enseignement s'est fait avec les signes utilisés par les humains sourdsmuets ; dans d'autres, il s'agissait d'associer des objets ou des actions à des lexigrammes. 
Au cours de ces expériences, les chimpanzés manifestent une capacité à pratiquer un langage symbolique. "Cependant, aussi spectaculaires soient-elles, les performances » des chimpanzés « ne sont pas assimilables aux compétences linguistiques » des humains : les combinaisons de lexigrammes sont beaucoup moins nombreuses et la presque totalité des symboles utilisés le sont « dans un contexte impératif, c'est-à-dire sur demande, alors que l'enfant utilise le langage dans un contexte informatif ou déclaratif », pour une communication de type référentiel. Enfin, l'apprentissage est beaucoup plus long ; dès l'âge de trois ans, le singe stagne alors que les enfants progressent de manière fulgurante. Mais surtout, on peut se demander si les capacités cognitives des grands singes révélées en laboratoire sont naturelles ou si elles résultent des interactions avec les expérimentateurs. L'environnement défini par l'homme en laboratoire jouerait-il un rôle dans l'émergence de certaines capacités cognitives, ces dernières n'ayant apparemment pas d'équivalent dans le répertoire de l'espèce dans la nature ?

Comme le remarque Lestel, le singe sur lequel on expérimente doit être considéré " comme un commensal de l'homme, c'est-à-dire profitant de l'homme pour survivre ". L'enseignement à travers lequel l'animal a accès aux rudiments d'un langage symbolique renvoie « à un phénomène de domestication mutuelle et cognitive » dans lequel les relations affectives jouent un rôle important. Il ne s'agirait pas véritablement de situation de communication, mais de test que l'homme propose aux singes. "Mais que peuvent-ils nous dire d'autre que ce que nous attendons d'eux? » Nous ignorons tout de leurs modes de communication symbolique dans la nature. En d'autres termes, « notre intelligence est-elle adéquate pour penser celle de l'animal » ? Il ne faut pas oublier que notre lignée a divergé de celle des chimpanzés il y a environ 7 millions d'années et que, depuis, chacun a évolué de son côté.

Cependant, ce que les observations dans la nature mettent en évidence, c'est l'importance de la communication entre les congénères, communication multimodale que l'on retrouve aussi chez l'homme, malgré la prééminence du langage. Ce qui fait alors la différence, c'est « l'aptitude à gérer la cognition de l'autre ». Les primates nonhumains ne savent pas utiliser les compétences et les savoir-faire de l'autre. Cependant, des expériences ont mis en évidence l'aptitude des chimpanzés à induire en erreur et à déjouer un partenaire humain qui tente de le tromper. Les chercheurs ont pu établir une corrélation entre la capacité à se reconnaître dans un miroir et à attri- buer à l'autre des états mentaux, ce dont sont capables les chimpanzés mais pas les macaques ; cette capacité est évidemment essentielle pour que s'instituent des interactions sociales. Les recherches tendant à déterminer comment a émergé la reconnaissance de soi dans la nature et ses liens avec l'utilisation d'outils ne sont guère convaincantes. Tout dépend de ce que l'on appelle reconnaissance de soi. Cyrulnik rappelle oportunément que la discrimination biologique du soi et du non-soi apparaît dès le début de l'histoire du vivant. Par contre, il insiste sur le saut que constitue, dans l'histoire de la conscience, l'émergence de la conscience réfléchie que seule la parole permet de partager.

De plus, cet auteur attire l'attention sur les conditions dans lesquelles se fait l'apprentissage cognitif et comment, pour les oiseaux, les mammifères et donc l'homme, "l'attachement » est nécessaire pour que se développent « les promesses génétiques ». Pour certaines espèces, le programme ontogénétique est presque directement inscrit dans le programme génétique. Pour de nombreuses autres espèces, dont les primates, la situation est différente : « le jeune se construit comportementalement et psychiquement par le biais d'interactions avec ses congénères et avec tout ce qui l'entoure » ; à travers son programme génétique, il n'hérite que de potentialités. Dans le monde animal, il faut distinguer les espèces à gestation courte, comme les rongeurs, où les petits naissent, dans un nid, avec les yeux et les oreilles fermées et une très faible mobilité, et les espèces dites « précoces » qui sont capables de se mouvoir quelques heures après leur naissance, comme les ongulés. Les primates non humains font partie des espèces précoces alors que l'homme, qui l'était aussi, aurait acquis secondairement des caractères d'immaturité à la naissance. En particulier, la croissance du cerveau se prolonge durant la première année de la vie postnatale, alors qu'elle se ralentit au cours du troisième mois chez les macaques. Il y a donc prolongation d'une caractéristique fœtale qui pourrait expliquer l'augmentation du nombre de neurones. En outre, naissant après une longue gestation, sa maturité sexuelle est également retardée. Or, " chez les primates, la myélinisation des structures cérébrales qui détermine la vitesse fonctionnelle des influx nerveux n'est achevée que vers l'adolescence... La longue maturation nerveuse peut être considérée comme spécifique des primates supérieurs ". Il est d'ailleurs curieux que la seule allusion au phénomène dit de " néoténie » soit le fait de la philosophe É. de Fontenay, dans le dernier chapitre. 


\section{La société précède-t-elle l'humanité ?}

Les aptitudes à la vie sociale sont traitées par $F$. De Waal et B. Thierry. Ils se sont en particulier intéressés à la résolution des conflits chez les singes : les attitudes de réconciliation, de consolation, les comportements de réassurance par contact corporel, les toilettages et épouillages. Il faut voir là « des dispositions positives à l'égard de la communauté » qui participent de la restauration de la vie sociale.

Les rapports de dominance et de soumission diffèrent notablement d'une espèce à l'autre, mais elles ne peuvent à elles seules expliquer le respect des règles ; " le désir d'appartenance au groupe [...] explique également que l'on se conforme à l'ordre établi ». Les rapports sociaux dépendent de la capacité des membres d'un groupe à coopérer et à échanger. Cependant, « l'équilibre entre intérêt personnel et intérêt collectif n'est pas situé au même point chez toutes les espèces ". Nous devons admettre avec les auteurs que « l'animal connaît le souci de la communauté, le sens de l'ordre social et l'intériorisation des normes. Dans cette perspective, le sens du bien et du mal s'est développé peu à peu au cours de l'évolution biologique ».

Les recherches des éthologues obligent à remettre en question des siècles de réflexion des philosophes sur « l'état de nature » et la conviction que celui-ci doit être reconstruit uniquement à partir de la psychologie des individus. Pour Rousseau, « la société n'a pu être que la mise en œuvre des propriétés caractéristiques de la nature individuelle [...] l'homme naturel est réduit aux seules sensations [...] il est asocial [...] la moralité n'a pu naître qu'avec la société » (Durkheim, 1953). Nous constatons au contraire que la vie en société et les relations que cela implique avec les congénères, l'empathie et le souci de la communauté, est un héritage de l'évolution biologique, et qu'au contraire c'est l'évolution culturelle qui nous a menés au règne de l'individualisme.

Comme le dit Godelier, (1984) « les hommes ne se contentent pas de vivre en société, ils produisent de la société pour vivre ". Grâce à la parole, de multiples «contrats sociaux » se sont peu à peu structurés, non pas à partir de la volonté consciente des individus, mais dans le cadre de cet héritage biologique qui fait de l'homme un être qui vit en relation avec d'autres. C'est ce principe qu'adopte L. Dumont (1983) pour définir la « sorte de sociologie » qu'il prône : « on part du fait que l'homme est un être social, on pose donc comme irréductible [...] le fait global de la société - non pas de la « société dans l'abstrait, mais chaque fois de telle société concrète particulière ». L'individu n'est pas un donné a priori, chacun se construit inséré dans un réseau de relations et d'échanges avec les membres de son groupe, mais aussi avec les éléments naturels du milieu.

La fameuse «énigme du don $»^{5}$, cette pratique qui veut que, lorsque l'on donne, on attend quelque chose en retour, et qui préoccupe tant les anthropologues, n'est-elle pas déjà inscrite dans les relations qu'entretiennent les anthropoïdes à l'intérieur du groupe dans lequel ils vivent ? Le langage parlé a permis que s'organisent des règles d'échanges entre les hommes, d'échanges avec des êtres de l'au-delà qu'ils imaginent détenir un pouvoir sur leurs moyens d'existence, règles le plus souvent vécues de façon implicite.

Le dernier mot sur "le propre de l'homme » revient à la philosophie. É. de Fontenay se demande s'il est légitime de le rechercher : «l'homme ne peut et ne doit être défini ». Elle convie Nietzsche pour affirmer que « l'homme [...] n'est absolument pas le couronnement de la création » et qu'il est « l'animal dont le caractère propre n'est pas encore fixé ». Elle conclut avec Merleau-Ponty que " l'on ne voit plus bien où commence le comportement et où finit l'esprit » et donne le mot de la fin à Marguerite Duras, qui dit à propos d'une gorille : « Nous sommes aussi séparés d'elle que du vide qui est devant nous ».

\section{Conclusion}

Que dire au terme de cette réflexion à propos de ces deux ouvrages?

Tout d'abord que l'on ne peut faire l'économie de suivre la chronique de nos origines depuis l'émergence de notre lignée, si nous voulons comprendre qui nous sommes. Les tâtonnements qu'elle révèle dans les différentes directions prises par l'évolution nous obligent à considérer avec beaucoup de modestie la place que nous occupons dans le monde vivant, et beaucoup de circonspection le sens qu'il faut donner à la conscience que nous avons de nous-mêmes et du monde. L'histoire de nos ancêtres est là aussi pour conforter les doutes qui se manifestent de plus en plus à l'égard du concept de "progrès ".

De cette chronique des origines, il ressort que nous sommes décidément bien seuls face à nos

\footnotetext{
${ }^{5}$ Cette expression est reprise du titre d'un ouvrage de $M$. Godelier (2002), mais le texte fondateur sur ce thème est le fameux «Essai sur le don » de M. Mauss (1923-1924).
} 
responsabilités, l'avenir de la planète. De notre ancrage dans l'évolution biologique n'émerge qu'une conscience nouvelle encore bien diluée et peu structurée d'une solidarité à l'égard des autres êtres vivants. Conscience nouvelle ou conscience retrouvée, car, dans le fonctionnement des sociétés humaines qui se sont succédé depuis que notre espèce a émergé, tout indique que cette solidarité, formalisée sous une forme ou une autre, a hanté l'humanité.

\section{Références}

Dumont, L., 1983. Essais sur l'individualisme. Une perspective anthropologique sur l'idéologie moderne. Seuil, Paris.

Durkheim, E., 1953. Montesquieu et Rousseau précurseurs de la sociologie. Librairie Marcel Rivière et Cie, Paris.

Godelier, M., 1984. L'idéel et le matériel. Pensée, économies, sociétés. Fayard, Paris.

Godelier, M., 2002. L'énigme du don. Flammarion, Paris.

Mauss, M., 1923-1924. Essai sur le don, forme et raison de l'échange dans les sociétés archaïques. Année Sociologique, seconde série tome 1 .

Available online at www.sciencedirect.com

Science $\mathcal{C}$ Directo 